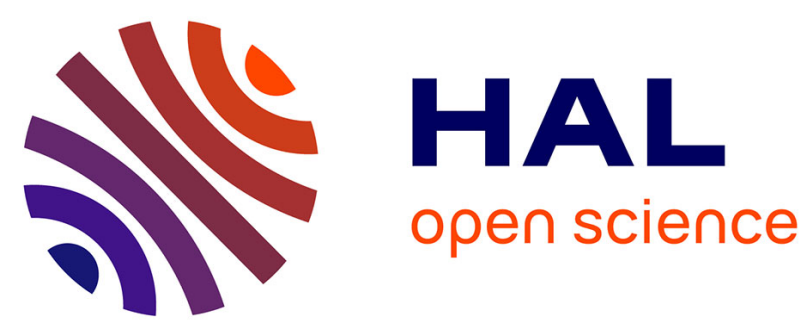

\title{
Analyse des nucléotides adényliques dans les feuilles de piment au cours d'une induction systémique de résistance à Phytophthora capsici L. et après une infection par ce même pathogène
}

Roselyne Lorient, Claude Coulomb, Alain Pradet

\section{To cite this version:}

Roselyne Lorient, Claude Coulomb, Alain Pradet. Analyse des nucléotides adényliques dans les feuilles de piment au cours d'une induction systémique de résistance à Phytophthora capsici L. et après une infection par ce même pathogène. Agronomie, 1987, 7 (10), pp.807-811. hal-00884956

\section{HAL Id: hal-00884956 https://hal.science/hal-00884956}

Submitted on 1 Jan 1987

HAL is a multi-disciplinary open access archive for the deposit and dissemination of scientific research documents, whether they are published or not. The documents may come from teaching and research institutions in France or abroad, or from public or private research centers.
L'archive ouverte pluridisciplinaire HAL, est destinée au dépôt et à la diffusion de documents scientifiques de niveau recherche, publiés ou non, émanant des établissements d'enseignement et de recherche français ou étrangers, des laboratoires publics ou privés. 


\title{
Analyse des nucléotides adényliques dans les feuilles de piment au cours d'une induction systémique de résistance à Phytophthora cap- sici L. et après une infection par ce même patho- gène
}

\author{
Roselyne LORIENT, Claude COULOMB \& Alain PRADET (*) \\ Faculté des Sciences. Laboratoire de Physiologie et Pathologie végétales, 33, rue Louis-Pasteur, F 84000 Avi- \\ gnon \\ (*) I.N.R.A., Station de Physiologie végétale, Centre de Recherches de Bordeaux, F 33140 Pont-de-la-Maye
}

RÉSUMÉ

L'analyse des nucléotides adényliques a été réalisée sur des feuilles de Capsicum annuum L., de variétés sensible et résistante, infectées par Phytophthora capsici ou élicitées par un inducteur systémique de résistance : le $\mathrm{G}_{1}$ TA. Les valeurs trouvées dans les plantes résistantes et sensibles sont à peu près identiques. Dans les feuilles de la variété sensible, l'élicitation et l'infection provoquent une augmentation significative de tous les paramètres, excepté pour l'ADP. Le rapport ATP/ADP double. Dans les feuilles de la variété résistante, la stimulation est moins importante et l'infection induit une diminution de chaque paramètre étudié.

Les résultats sont discutés en fonction de leur implication possible dans les mécanismes de résistance et de sensibilité en réponse à l'infection ou à l'élicitation.

Mots clés additionnels : Phytopathologie, potentiel énergétique, facteurs de résistance. Phytophthora capsici $L$. and after inoculation with this pathogen.

\begin{abstract}
Adenine nucleotide analysis was performed on susceptible and resistant leaves of Capsicum annuum L. infected by Phytophthora capsici $\mathrm{L}$. or induced by a systemic resistance elicitor $\mathrm{G}_{1} \mathrm{TA}$. The values found in resistant and susceptible plants were similar. In susceptible leaves, elicitation and infection provoked a significant increase of all parameters, except for ADP. The ATP/ADP ratio doubled. In resistant leaves, the stimulation was lower and the infection induced a decrease of each parameter studied. The results are discussed with respect 10 their hypothetical involvement in the character of resistance and susceptibility to infection and elicitation.
\end{abstract}

Additional key words : Phytopathology, energy charge, defense mechanisms.

\section{INTRODUCTION}

La mesure de l'ATP et de ses précurseurs donne des informations importantes sur l'activité métabolique des organismes car les nucléotides adényliques sont intégrés dans un système de régulation extrêmement complexe qui permet d'adapter la production de l'énergie aux besoins de la cellule. Il est donc intéressant de connaître le comportement des nucléotides adényliques des feuilles de piment de variétés sensible et résistante au cours d'une induction systémique de résistance au Phytophthora capsici ou après une infection par ce même pathogène.

Les travaux réalisés ces dernières années sur ce matériel ont permis de révéler l'apparition d'une résistance de l'ordre de 80 p. 100 au niveau des feuilles lorsqu'une induction a été préalablement réalisée au niveau des racines (CoulomB C. et al., 1983). Cette induction non spécifique ne s'accompagne pas de production de Capsidiol, phytoalexine de nature sesquiter- 
pénique synthétisée par le piment (STOESSL, 1982 ; MOLOT et al., 1984) mais entraîne une augmentation de la concentration en protéines totales parmi lesquelles certaines fractions présentent une activité peroxydasique décuplée; les activités phénylalanine ammonium lyase et catalase sont également stimulées (COULOMB C., résultats non publiés).

Après contamination par le Phytophthora capsici l'activité peroxydase augmente également dans les feuilles des deux variétés mais davantage chez la variété résistante où de nouvelles formes moléculaires apparaissent (COULOMB C. et Ph., 1984).

Enfin LORIENT \& BOUNIAS (1985) ont noté une augmentation significative de la teneur en glucides dans les cellules voisines des assises cellulaires nécrotiques concernées par la réaction d'hypersensibilité chez la variété résistante.

Tous ces processus exigent une forte dépense d'énergie soit pour compenser les prélèvements effectués par le parasite, soit pour assurer la mise en œuvre des moyens de défense. Dès lors, c'est en fonction des potentialités génétiques de l'hôte à intensifier la production cellulaire d'énergie nécessaire à sa défense que se manifestera l'efficacité de sa réponse. Cette énergie est emmagasinée sous forme d'ATP puis communiquée aux sites biosynthétiques pour y être réutilisée.

\section{MATÉRIEL ET MÉTHODE}

\section{A. Matériel}

Le matériel végétal comporte 2 variétés de piment (C. annuum) dont l'une est sensible (Yolo Wonder) et l'autre résistante (Phyo 636) à un champignon pathogène, le $P$. capsici, responsable d'une maladie de type « mildiou ».

- Les expériences d'induction de résistance (élicitation) sont réalisées sur des plantes âgées d'un mois et cultivées en terrines sur un mélange de tourbe et de terreau désinfecté à la vapeur. Le système racinaire des plantules est soigneusement rincé, essuyé puis immergé pendant $48 \mathrm{~h}$ dans la solution élicitrice ou dans de l'eau pour les témoins. La solution élicitrice $\mathrm{G}_{1} \mathrm{TA}$ est préparée selon un protocole décrit par MOLOT et al. (1980), à partir du milieu de culture de Trichoderma album. L'induction de résistance est appréciée au niveau des feuilles par dépôt d'une suspension de zoospores (MOLOT et al. (1980)).

- Les infections sont réalisées sur des plantes âgées de 2 mois repiquées au stade des deux cotylédons, en utilisant le mycélium de $P$. capsici, souche $15 \mathrm{~A}$, âgé de 8 jours et cultivé en boîtes de Petri sur le milieu L de MESSIAEN \& LAFON (1970).

Des pastilles de mycélium de $4 \mathrm{~mm}$ de diamètre sont déposées au centre de la face supérieure des feuilles préalablement blessées à l'aide d'une aiguille. Ces manipulations sont réalisées dans des conditions aseptiques. Les plantes inoculées sont entourées d'une enveloppe plastique qui évite la dessication et disposées en chambre climatisée $\left(22^{\circ} \mathrm{C}\right.$ éclairée $10 \mathrm{~h}$ par jour $8000 \mathrm{lux}, 38.4 .10 \mathrm{erg} / \mathrm{cm} \mathrm{sec}$ ).

Après 3 jours d'infection, les dosages sont réalisés sur les tissus foliaires prélevés en dehors de la zone de nécrose.

\section{B. Dosage des nucléotides adényliques}

Le dosage enzymatique des nucléotides adényliques est réalisé selon la technique de la bioluminescence mise au point par PRADET (1967) puis SAGLIo et al. (1979). L'oxydation de la luciférine est la source d'énergie de l'émission de lumière. La vitesse de cette oxydation est proportionnelle à la concentration du milieu en ATP. Le dosage de l'ATP se ramène donc à la mesure de l'intensité lumineuse observée lorsqu'on met en présence un extrait végétal contenant ce composé avec une solution de luciférine. A la fin du temps d'incubation, les feuilles sont immédiatement prélevées, pesées et plongées dans l'azote liquide ; la fixation et l'extraction ont lieu à $0{ }^{\circ} \mathrm{C}$ en présence d'acide trichloracétique ( 5 p. $100 \mathrm{~W} / \mathrm{V})$ qui est ensuite éliminé par une extraction à l'éther. Les échantillons sont stockés à $-25^{\circ} \mathrm{C}$. Les dosages sont effectués à l'aide d'un biophotomètre Pico-ATP (JOBIN \& YvON). Le signal lumineux est transformé en signal électrique (enregistreur SEFRAM).

La hauteur des pics est calculée à l'aide d'un intégrateur Icap 80. Les valeurs obtenues sont comparées à celles des étalons et la quantité d'ATP est calculée après programmation (ordinateur PSI 80 Kontron).

L'ADP et l'AMP sont transformés en ATP en présence de pyruvate kinase et d'adénylate kinase.

En effectuant les trois dosages successivement et en opérant par différence, on obtient la teneur de la solution en AMP, ADP et ATP. Les résultats sont exprimés en nanomoles/g de matière fraîche.

\section{RÉSULTATS}

\section{A. Influence d'une induction de résistance}

L'analyse des nucléotides adényliques a été pratiquée sur les feuilles de plantules âgées d'un mois exposées à la lumière et d'autres maintenues à l'obscurité. Les résultats (tabl. 1) montrent que chez les plants témoins, sensibles et résistants les quantités d'ATP et d'ADP, et les valeurs de la somme des nucléotides sont très voisines. Ces valeurs sont plus faibles (de 30 à 40 p. 100) lorsque l'analyse est pratiquée sur des plants maintenus à l'obscurité.

L'élicitation provoque chez les plantules des 2 variétés, qu'elles soient exposées à la lumière ou maintenues à l'obscurité, une augmentation significative des valeurs de la quantité d'ATP, de la somme des nucléotides et de la somme des liaisons riches en énergie. Le rapport ATP/ADP double chez les plantes élicitées de la variété sensible alors que chez la variété résistante, il ne s'accroît que de 30 p. 100 de sa valeur. Seules les quantités d'ADP restent stables.

Sous l'action de l'éliciteur et pour des plantes maintenues à la lumière, les différents paramètres étudiés traduisent une stimulation plus significative pour ceux de la variété sensible que pour ceux de la variété résistante. 
TABLEAU 1

Action de l'élicitation par le $G_{I} T A$ sur les concentrations en nucléotides adényliques des feuilles de Capsicum annuum de variétés sensible (Yolo Wonder) et résistante (Phyo 636) (plants âgés de l mois).

Les quantités de nucléotides sont exprimées en nanomoles par $\mathrm{g}$ de matière fraîche. Les moyennes $(x)$ et les écarts types $\left(S_{X}\right)$ ont été calculés à partir de 3 échantillons indépendants.

Action of the elicitor $G, T A$ on adenine nucleotide concentration in susceptible (Yolo Wonder) and resistant (Phyo 636) leaves of 1 month old plants of Capsicum annuum.

Nucleotides values expressed in nmole per $g$ fresh weight. Means and standard deviation calculated from three independent samples.

\begin{tabular}{|c|c|c|c|c|c|c|c|c|c|}
\hline \multirow[b]{3}{*}{ Paramètres étudiés } & \multirow[t]{3}{*}{ Variétés } & \multicolumn{4}{|c|}{ Yolo Wonder } & \multicolumn{4}{|c|}{ Phyo 636} \\
\hline & & \multicolumn{2}{|c|}{ Témoin } & \multicolumn{2}{|c|}{ Elicité } & \multicolumn{2}{|c|}{ Témoin } & \multicolumn{2}{|c|}{ Elicité } \\
\hline & & Lumière & Obscurité & Lumière & Obscurité & Lumière & Obscurité & Lumière & Obscurité \\
\hline Somme des nucléotides & $\begin{array}{l}\mathrm{X} \\
\mathrm{S}_{\mathrm{X}}\end{array}$ & $\begin{array}{l}99 \\
11,5\end{array}$ & $\begin{array}{l}71 \\
6,8\end{array}$ & $\begin{array}{r}158 \\
17\end{array}$ & $\begin{array}{l}88 \\
9,7\end{array}$ & $\begin{array}{l}98 \\
4,3\end{array}$ & $\begin{array}{r}63 \\
5\end{array}$ & $\begin{array}{l}130 \\
12,5\end{array}$ & $\begin{array}{l}95 \\
12,4\end{array}$ \\
\hline ATP & $\begin{array}{l}\mathrm{X} \\
\mathrm{S}_{\mathrm{X}}\end{array}$ & $\begin{array}{l}72 \\
10\end{array}$ & $\begin{array}{l}46 \\
3,2\end{array}$ & $\begin{array}{r}125 \\
19\end{array}$ & $\begin{array}{l}65 \\
7,8\end{array}$ & $\begin{array}{l}67 \\
3,4\end{array}$ & $\begin{array}{l}40 \\
2,3\end{array}$ & $\begin{array}{r}100 \\
16\end{array}$ & $\begin{array}{l}70 \\
10,6\end{array}$ \\
\hline $\mathrm{ADP}$ & $\begin{array}{l}\mathrm{X} \\
\mathrm{S}_{\mathrm{X}}\end{array}$ & $\begin{array}{l}20 \\
2,4\end{array}$ & $\begin{array}{r}19 \\
3\end{array}$ & $\begin{array}{l}20 \\
6,4\end{array}$ & $\begin{array}{l}17 \\
1,8\end{array}$ & $\begin{array}{r}24 \\
3\end{array}$ & $\begin{array}{l}18 \\
2,5\end{array}$ & $\begin{array}{r}27 \\
7\end{array}$ & $\begin{array}{l}18 \\
2,3\end{array}$ \\
\hline $\mathrm{ATP} / \mathrm{ADP}$ & & 3,6 & 2,4 & 6,25 & 3,8 & 2,8 & 2,2 & 3,7 & 3,8 \\
\hline $\begin{array}{l}\text { Somme des liaisons riches } \\
\text { en énergie }\end{array}$ & $\begin{array}{l}\mathrm{X} \\
\mathrm{S}_{\mathrm{X}}\end{array}$ & $\begin{array}{r}164 \\
21\end{array}$ & $\begin{array}{l}112 \\
8,4\end{array}$ & $\begin{array}{r}274 \\
37\end{array}$ & $\begin{array}{r}146 \\
15\end{array}$ & $\begin{array}{r}160 \\
5,8\end{array}$ & $\begin{array}{r}100 \\
6,7\end{array}$ & $\begin{array}{r}225 \\
2,7\end{array}$ & $\begin{array}{l}160 \\
21,6\end{array}$ \\
\hline
\end{tabular}

\section{B. Influence de la contamination}

L'analyse des nucléotides adényliques a été pratiquée sur des feuilles saines et contaminées de plants de piment âgés de 2 mois et exposés ou non à la lumière. Les prélèvements d'échantillons sur les feuilles contaminées sont toujours réalisés en dehors de la zone nécrosée (tabl. 2).

Chez les plants sains, aucune différence significative n'est observée ni à la lumière, ni à l'obscurité entre les valeurs initiales des teneurs en nucléotides des 2 variétés. Lors de la transition de la phase éclairée à la phase obscure une certaine diminution est observée dans les 2 cas. Cependant, les différences sont faibles ( -10 à - 16 p. 100) et non significatives dans le cas de la variété sensible alors que dans la variété résistante l'écart est plus marqué (de -30 à $-40 \mathrm{p}$. 100) et toujours significatif.

Après 3 jours de contamination une importante différence apparaît entre les 2 variétés.

Chez les plantes sensibles éclairées les teneurs en ATP augmentent de 48 p. 100 . Les augmentations observées dans le cas de la somme des nucléotides et de celle des liaisons riches en énergie sont moins impor-

TABLEAU 2

Action de la contamination par le mildiou sur les concentrations en nucléotides adényliques des feuilles saines et contaminées de Capsicum annuum, de variétés sensible (Yolo Wonder) et résistante (Phyo 636) (plants âgés de 2 mois).

Les quantités de nucléotides sont exprimées en nanomoles par $\mathrm{g}$ de matière fraîche. Les moyennes $(X)$ et les écarts types $\left(S_{X}\right)$ ont été calculés à partir de 3 échantillons indépendants.

Action of Phytophthora capsici infection on adenylic nucleotide concentration in susceptible (Yolo Wonder) and resistant (Phyo 636) leaves of 2 month old plants of Capsicum annuum.

Nucleotide values expressed in nmole per $g$ fresh weight. Means and standard deviation calculated from three independent samples.

\begin{tabular}{|c|c|c|c|c|c|c|c|c|c|}
\hline \multirow[b]{3}{*}{ Paramètres étudiés } & \multirow[t]{3}{*}{ Variétés } & \multicolumn{4}{|c|}{ Yolo Wonder } & \multicolumn{4}{|c|}{ Phyo 636} \\
\hline & & \multicolumn{2}{|c|}{ Témoin } & \multicolumn{2}{|c|}{ Contaminé } & \multicolumn{2}{|c|}{ Témoin } & \multicolumn{2}{|c|}{ Contaminé } \\
\hline & & Lumière & Obscurité & Lumière & Obscurité & Lumière & Obscurité & Lumière & Obscurité \\
\hline Somme des nucléotides & $\begin{array}{l}\mathrm{X} \\
\mathrm{S}_{\mathrm{X}}\end{array}$ & $\begin{array}{l}228 \\
(31,7)\end{array}$ & $\begin{array}{l}202 \\
(22)\end{array}$ & $\begin{array}{l}267 \\
(48)\end{array}$ & $\begin{array}{l}228 \\
(48)\end{array}$ & $\begin{array}{l}257 \\
(34)\end{array}$ & $\begin{array}{l}166 \\
(22)\end{array}$ & $\begin{array}{l}167 \\
(28)\end{array}$ & $\begin{array}{l}114 \\
(8,6)\end{array}$ \\
\hline ATP & $\begin{array}{l}\mathrm{X} \\
\mathrm{S}_{\mathrm{X}}\end{array}$ & $\begin{array}{l}172 \\
(28)\end{array}$ & $\begin{array}{l}155 \\
(18)\end{array}$ & $\begin{array}{l}255 \\
(40)\end{array}$ & $\begin{array}{l}194 \\
(52)\end{array}$ & $\begin{array}{l}186 \\
(26)\end{array}$ & $\begin{array}{l}127 \\
(18)\end{array}$ & $\begin{array}{l}129 \\
(23)\end{array}$ & $\begin{array}{l}85 \\
(7)\end{array}$ \\
\hline ADP & $\begin{array}{l}\mathrm{X} \\
\mathrm{S}_{\mathrm{X}}\end{array}$ & $\begin{array}{l}48 \\
(5)\end{array}$ & $\begin{array}{l}40 \\
(4)\end{array}$ & $\begin{array}{l}35 \\
(7)\end{array}$ & $\begin{array}{l}29 \\
(9)\end{array}$ & $\begin{array}{l}60 \\
(8)\end{array}$ & $\begin{array}{l}37 \\
(4)\end{array}$ & $\begin{array}{l}28 \\
(5)\end{array}$ & $\begin{array}{l}27 \\
(2)\end{array}$ \\
\hline ATP/ADP & & 3,5 & 3,8 & 6,5 & 7,5 & 3,1 & 3,4 & 4,6 & 3,1 \\
\hline $\begin{array}{l}\text { Somme des liaisons riches } \\
\text { en énergie }\end{array}$ & $\begin{array}{l}\mathrm{X} \\
\mathrm{S}_{\mathrm{X}}\end{array}$ & $\begin{array}{l}392 \\
(62)\end{array}$ & $\begin{array}{l}352 \\
(41)\end{array}$ & $\begin{array}{l}486 \\
(85)\end{array}$ & $\begin{array}{l}419 \\
(100)\end{array}$ & $\begin{array}{l}433 \\
(59)\end{array}$ & $\begin{array}{l}291 \\
(40)\end{array}$ & $\begin{array}{l}287 \\
(50)\end{array}$ & $\begin{array}{l}198 \\
(50)\end{array}$ \\
\hline
\end{tabular}


tantes de même que toutes celles mesurées en phase obscure.

L'ADP diminue de façon peu significative aussi bien à la lumière qu'à l'obscurité où le rapport ATP/ADP augmente respectivement de 86 et 97 p. 100.

Dans le cas de la variété résistante les concentrations de tous les nucléotides diminuent très significativement ( -27 à -35 p. 100) et plus particulièrement l'ADP en phase éclairée. Par contre le rapport ATP/ADP varie dans de moindres proportions : augmentation de 48 p. 100 à la lumière et diminution de 9 p. 100 à l'obscurité.

\section{DISCUSSION}

Les résultats montrent en premier lieu que les différences de composition en nucléotides entre la variété sensible et la variété résistante se manifestent sous la pression des phénomènes physiopathologiques que sont l'élicitation et l'infection.

Dans les conditions expérimentales observées les conséquences de l'élicitation présentent de grandes analogies avec celles de l'infection chez la variété sensible alors qu'elles divergent totalement dans les feuilles de la variété résistante. Dans le premier cas, toutefois, l'augmentation des nucléotides n'est significative après contamination, que dans les plants éclairés, alors qu'elle est marquée dans tous les cas (quoique plus significativement à la lumière) sous l'action de l'éliciteur.

L'éliciteur prépare donc les plants sensibles à affronter une situation traumatique en élevant leur potentiel énergétique, tandis que dans le cas de l'infection ce même potentiel paraît déjà entamé. Dans les plantes de la variété résistante ce même phénomène apparaît avec davantage de netteté : l'élicitation accroît simplement le potentiel énergétique alors qu'après infection celui-ci est fortement entamé très vraisemblablement du fait d'une utilisation considérablement plus intensive de l'ATP dans la biosynthèse des divers facteurs de résistance.

A notre connaissance aucun autre travail n'a été à ce jour consacré à l'étude de l'influence de l'élicitation sur les systèmes générateurs d'énergie ; par contre dans les tissus blessés de pomme de terre, TAKAMURA \& URITANI (1973) ont observé une augmentation de la teneur en nucléotides. On sait par ailleurs, qu'en général l'infection provoque une augmentation substantielle de l'activité respiratoire (URITANI \& ASAKI, 1980) et une baisse de l'activité photosynthétique (MOBTALBINI \& BUCHANAN, 1974 ; MAgYAROSY \& MALKIN, 1978). La phosphorylation oxydative et la synthèse des ARN peuvent être stimulées (HUTCHESON \& BUCHANAN, 1983) ainsi que les nombreux systèmes enzymatiques associés à la biosynthèse des composés phénoliques, des lignines et des phytoalexines (FRICK, 1976 ; SIMONS \& ROSS, 1970 ; HAMMERSCHMIDT et al., 1972 : PAYNOT, 1976).

GIANNINAZZI et al. (1980); AHL et al. (1982); DUMAS \& GIANNINAZZI (1986) ont signalé puis discuté l'importance de l'apparition de nouvelles protéines de type $b$ dans la résistance aux virus et aux champignons. Ces manifestations s'ajoutent à celles déjà recensées chez les plantes induites ou infectées : à savoir l'accumulation des phytoalexines (DEVERALL, 1977 ; CALLOW, 1977 ; MANSFIELD, 1982) et de dérivés phénoliques toxiques (KoSUGE, 1969) ou encore l'enrichissement des parois de l'hôte en lignines (TOUzE \& ROSSIGNOL, 1976-1980 : SHERWOOD \& VANCE, 1980) ; (HAMMERSCHMIDT \& KUC, 1982) ou en glycoprotéines à hydroxyproline (ESQUERRE-TUGAYE et al., 1979).

Les nucléotides fournissent l'énergie consommée par toutes ces voies métaboliques, les fluctuations que nous observons témoignent de leur utilisation différencielle au sein de tissus induits ou infectés.

Recu le 23 avril 1986 Accepté le 18 juillet 1987.

\section{RÉFÉRENCES BIBLIOGRAPHIQUES}

Ahl P., Cornu A., Gianinazzi S., 1982. Soluble proteins as genetic markers in studies of resistance and phylogeny in Nicotiana. Phytopathology, 72, 80-85.

Callow J. A., 1977. Recognition, resistance and the role of plant lectins in host-parasite interactions. $A d v$. Bot. Res., 4, 2-50.

Coulomb C., Molot M., Mas P., Coulomb Ph., Bounias M., 1983. Analyse des variations de l'activité péroxydasique soluble (E.C. 1.11.1.7.) dans les feuilles de Capsicum annumm (Piment) au cours d'une induction systémique de résistance au Phytophthora capsici (Mildiou). C. R. Acad. Sci., 296, sér. 11I, 225-230.

Coulomb C. et Coulomb Ph. J., 1984. Etude de l'activité péroxydasique dans les feuilles de Capsicum annuum (piment) infectées par le Phytophthora capsici (Mildiou). Ann. Sci. Nat., 6, 227-235.

Deverall B. J., 1977. Defence mechanisms of plants. Cambr. Univ. Press. 110 p.

Dumas E., Gianninazzi S., 1986. Pathogenesis-related (b) proteins do not play a central rôle in TMV localization in Nicotiana rusíca. Physiol. Molec. Plant Pathol., 28, 243-250.
Esquerre Tugaye M. T., Lafitte C., Mazaud D., Toppan A., Touze A., 1979. Cell surfaces in plant-microorganism interactions. II. Evidence for the accumulation of hydroxyproline-rich glycoproteins in the cell wall of diseased plant as a defence mechanism. Plant Physiol., 64, 320-326.

Esquerre-Tugaye M. T., Mazaud D., Toppan A., Roby D., 1980. Elicitation via l'éthylène de la synthèse de glycoprotéines pariétales associées à la défense des plantes. Ann. Phytopathol., 12 (4), 403-411.

Frick F., 1976. Oxidative enzymes, 617-631. In Heitfuss R. \& William P. H. "Encyclopedia of Plant physiology », vol. IV, SpringerVerlag. New York.

Gianninazzi S., Ahl P., Cornu A., Scalla R., Cassini R., 1980. First report of host $\mathbf{b}$-protein appearance in response to a fungal infection in tobacco. Physiol. Plant Pathol., 16, 337-342.

Hammerschmidt R., Kuc J., 1982. Lignification as a mechanism for induced systemic resistance in cucumber. Physiol. Plant Pathol., 20, $61-71$. 
Hammerschmidt R., Nuckles E. M., Kuc J., 1982. Association of enhanced peroxidase activity with induced systemic resistance of cucumber to Colletotrichum lagenarium. Physiol. Plant Pathol., 20, 73-82.

Hutcheson S. W., Buchanan B. B., 1983. Bioenergic and metabolic disturbances in diseased plants. Bioch. Plant Pathol. Edited by J. A. Callow, 327-245.

Kosuge T., 1969. The role of phenolics in host response to infection. Annu. Rev. Phytopathol., 7, 195-222.

Lorient R., 1984. Contribution à l'étude de l'interaction Capsicum annuum-Phytophthora capsici. Action d'un fongicide de synthèse l'éthyl phosphite d'aluminium. Thèse $3^{\mathrm{e}}$ cycle. Université d'Aix Marseille II, $92 \mathrm{p}$.

Lorient R., Bounias M., 1985. Variations de la teneur en sucres solubles dans les feuilles de Piment Capsicum annuum infectées par P. capsici et traitées par le phosétyl d'aluminium. Fungicides for crop protection BCPC monograph $n^{\circ} 31$.

Magyarosy A. C., Malkin R., 1978. Effect of powdery mildew infection of sugar beet on the content of electron carriers in chloroplasts. Physiol. Plant Pathol., 13, 183-188.

Mansfield J. W., 1982. The role of phytoalexins in disease resistance, 253-258. In Ed. Bailey J. A. \& Mansfield J. W. "Phytoalexins ", $223 \mathrm{p}$.

Messiaen C. M., Lafon R., 1970. Les maladies des plantes maraîchères, 387, 388. I.N.R.A., Paris.

Molot P. M., Staron T., Mas P., 1980. La résistance du piment à Phytophthora capsici. VIII. Induction de résistance et de capsidiol chez Capsicum annuum avec des fractions obtenues à partir des filtrats de culture et du mycélium de Phytophthora capsici. Ann. Phytopathol., 12 (4) 379-387.

Molot P. M., Mas P., Conus M., Ferrière, 1984. La résistance du piment à Phytophthora capsici. XII. Influence de l'élicitation sur la résistance induite et l'accumulation de Capsidiol. Agronomie, 4 (9), 829-833.
Montalbini P., Buchanan B. B., 1974. Effect of a rust infection on photophosphorylation by isolated chloroplast. Physiol. Plant Pathol., 4, 191-196.

Paynot M., 1976. Etude d'une enzyme végétale la phénylalanine ammonium lyase en relation avec la réaction d'hypersensibilité du tabac au virus de la mosä́que du tabac. Thèse Doct. Etat Univ. de Dijon, 129 p.

Pradet A., 1967. Etude des adénosine-5' mono-di et triphosphates dans les tissus végétaux. I. Dosage enzymatique. Physiol. Vég., 5, 209-221.

Saglio Ph., Daniels M. J., Pradet A., 1979. ATP and energy charge as criteria of growth and metabolic activity of Mollicutes : application to Spiroplasma citri. J. Gen. Microbiol., 110, 13-20.

Sherwood R. T., Vance C. P., 1980. Resistance to fungal penetration in Graminaeae. Phytopathology, 70, 273-279.

Simons Th. J., Ross A. F., 1970. Enhanced peroxidase activity associated with induction of resistance to tobacco mosaic virus in hypersensitive tobacco. Phytopathology, 60, 383-384.

Stoessl A., 1982. Biosynthesis of phytoalexins. In «Phytoalexins. " Blackie, London, Glasgow, 133-180.

Takamura T., Uritani I., 1973. Changes in acid-soluble nucleotides in cut injured sweet potato root tissue. Agr. Biol. Chem., 37, 15111515.

Touzé A., Rossignol M., 1976. Lignification and the onset of premunition in muskmelon plants. In Solheim \& Raa «Cell wall biochemistry related to specificity in Host-Plant pathogen interactions ». Universitetsfor laget, Oslo, Norway, 289-293.

Touzé A., Rossignol M., 1980. La protection biologique des plantes contre les infections bactériennes et fongiques. Ann. Phytopathol., 12 (4), 379-387.

Uritani I., Asaki T., 1980. Respiration and related metabolic activity in wounded and infected tissues. In : " The Biochemistry of plants", vol. 2, Acad. Press, 463-483. 